\title{
Spinal cord ischemia after simultaneous and sequential treatment of multilevel aortic disease
}

\author{
Gabriele Piffaretti, MD, PhD, ${ }^{\mathrm{a}}$ Stefano Bonardelli, MD, ${ }^{\mathrm{c}}$ Raffaello Bellosta, MD, ${ }^{\mathrm{d}}$ \\ Giovanni Mariscalco, MD, PhD, ${ }^{\mathrm{b}}$ Chiara Lomazzi, MD, ${ }^{\mathrm{e}}$ Jip L. Tolenaar, MD, ${ }^{\mathrm{e}}$ Camilla Zanotti, MD, \\ Cristina Guadrini, MD, ${ }^{\mathrm{c}}$ Antonio Sarcina, MD, ${ }^{\mathrm{d}}$ Patrizio Castelli, MD, FACS, ${ }^{\mathrm{a}}$ and Santi Trimarchi, MD, PhD
}

Objectives: The aim of the present study is to report a risk analysis for spinal cord injury in a recent cohort of patients with simultaneous and sequential treatment of multilevel aortic disease.

Methods: We performed a multicenter study with a retrospective data analysis. Simultaneous treatment refers to descending thoracic and infrarenal aortic lesions treated during the same operation, and sequential treatment refers to separate operations. All descending replacements were managed with endovascular repair.

\begin{abstract}
Results: Of 4320 patients, multilevel aortic disease was detected in 77 (1.8\%). Simultaneous repair was performed in 32 patients $(41.5 \%)$, and a sequential repair was performed in 45 patients $(58.4 \%)$. Postoperative spinal cord injury developed in 6 patients $(7.8 \%)$. At multivariable analysis, the distance of the distal aortic neck from the celiac trunk was the only independent predictor of postoperative spinal cord injury (odds ratio, 0.75; $95 \%$ confidence interval, $0.56-0.99 ; P=.046)$; open surgical repair of the abdominal aortic disease was associated with a higher risk of spinal cord injury but did not reach statistical significance (odds ratio, 0.16; $95 \%$ confidence interval, 0.02-1.06; $P=.057$ ). Actuarial survival estimates at 1,2 , and 5 years after the procedure were $80 \% \pm 5 \%, 68 \% \pm 6 \%$, and $63 \% \pm 7 \%$, respectively. Spinal cord injury did not impair survival $(P=.885)$.
\end{abstract}

Conclusions: In our experience, the risk of spinal cord injury is still substantial at $8 \%$ in patients with multilevel aortic disease. The distance of the distal landing zone from the celiac trunk is a significant predictor of spinal cord ischemia. (J Thorac Cardiovasc Surg 2014;148:1435-42)

\section{Supplemental material is available online.}

Multilevel aortic disease (MAD) is an uncommon finding that still poses a treatment dilemma for cardiovascular surgeons. ${ }^{1-3}$ Simultaneous treatment has been associated with severe perioperative complications, whereas sequential repair requires 2 major surgical interventions, and rupture of the residual lesion can occur while waiting for the second operation. ${ }^{1,4}$ Regardless of the strategy

\footnotetext{
$\overline{\text { From Vascular }}$ Surgery $^{\mathrm{a}}$ and Cardiac Surgery, ${ }^{\mathrm{b}}$ Department of Surgery and Morphological Sciences Circolo University Hospital, University of Insubria School of Medicine, Varese, Italy; Vascular Surgery, ${ }^{\text {c }}$ Department of Surgery, Spedali Civili Hospital, University of Brescia School of Medicine, Brescia, Italy; Vascular Surgery, ${ }^{\mathrm{d}}$ Cardiovascular Department, Poliambulanza Foundation,

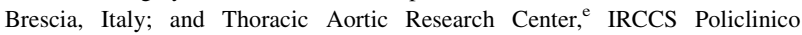
San Donato, San Donato Milanese, Milan, Italy.

Disclosures: Authors have nothing to disclose with regard to commercial support.

Received for publication Nov 28, 2013; revisions received Feb 6, 2014; accepted for publication Feb 21, 2014; available ahead of print March 31, 2014.

Address for reprints: Gabriele Piffaretti, MD, PhD, Vascular Surgery, Department of Surgery and Morphological Sciences, Circolo University Hospital, University of Insubria School of Medicine, Via Guicciardini 9, 21100 Varese, Italy (E-mail: gabriele.piffaretti@uninsubria.it).

$0022-5223 / \$ 36.00$

Copyright (C) 2014 by The American Association for Thoracic Surgery

http://dx.doi.org/10.1016/j.jtcvs.2014.02.062
}

adopted, these patients require extensive aortic treatment and are at high risk for spinal cord injury (SCI).

$\mathrm{Up}$ to now, definitive recommendations about the appropriate treatment are lacking. The rate of SCI after thoracic endovascular repair (TEVAR) has been reported as significantly higher with a history of abdominal aortic repair, but there are few data regarding the comparison of simultaneous versus sequential treatment of MAD. ${ }^{5-10}$

The aim of the present study is to report a risk analysis for SCI in a recent cohort of patients with simultaneous or sequential MAD treatment.

\section{MATERIALS AND METHODS \\ Patient Population}

Between October 2001 and March 2013, all patients undergoing simultaneous or sequential repair of separate descending thoracic and infrarenal abdominal aortic lesions were identified and included in the analysis. Patients with ascending aortic disease, thoracoabdominal extent, or mega-aortic syndrome were excluded. This is a retrospective multicenter study. All clinical and procedural data were prospectively collected and recorded in a dedicated computerized database that remained consistent over the study period. The authors had full access to and take full responsibility for the integrity of the data. The study protocol was in compliance with the local institutional review board and received full approval.

\section{Patient Management}

All patients underwent preoperative total aortic computed tomography angiography. The risk of SCI was debated case by case, and the type of 


\section{Abbreviations and Acronyms \\ ASIA = American Spinal Injury Association \\ CI = confidence interval \\ CSFD = cerebrospinal fluid drainage \\ LSA $=$ left subclavian artery \\ MAD $=$ multilevel aortic disease \\ $\mathrm{OR} \quad=$ odds ratio \\ SCI $=$ spinal cord injury \\ TEVAR $=$ thoracic endovascular repair}

treatment was discussed and approved collegially by the operating team. All patients gave their consent to participate. All descending thoracic treatments were TEVAR. When open abdominal aortic repair was preferred, it was performed via a midline laparotomy and transperitoneal approach. When simultaneous repair was performed with an endograft in both aortic areas, TEVAR was the first procedure of the operation. When abdominal repair was performed with an open approach (hybrid intervention) during simultaneous repair, TEVAR was performed after infrarenal graft replacement. When it was planned to cover the left subclavian artery (LSA), adjunctive prophylactic revascularization was performed in selected cases at higher risk for SCI (aortic coverage: extent type $\mathrm{C}$ or $>20 \mathrm{~cm}$ plus previous abdominal aortic repair in sequential cases), in those presenting with coronary circulation supplied by the LSA through the left internal thoracic artery, when there was a necessity to cover the origin of the left LSA with hypoplastic contralateral vertebral artery, in left-handed professionals, and in those with left arm arteriovenous fistula for dialysis. Cerebrospinal fluid drainage (CSFD) (Duet; Medtronic Vascular, Santa Rosa, Calif) was not placed on a routine basis but selectively: extensive aortic coverage (entire descending aorta, thoracic coverage $>20 \mathrm{~cm}$, thoracic coverage $<20 \mathrm{~cm}$ with involvement of $\mathrm{T}_{8}$ ) or when the LSA was scheduled to be overstented. ${ }^{11}$ Volume expansion or vasopressors were used to maintain mean arterial pressure greater than $75 \mathrm{~mm} \mathrm{Hg} .{ }^{11}$ Perioperative homologous packed red blood cells were administered to optimize hemoglobin concentration greater than $9 \mathrm{~g} / \mathrm{dL}$. In patients with CSFD, cerebrovascular pressure was monitored continuously in the perioperative period to achieve a target level of 10 to $12 \mathrm{~mm} \mathrm{Hg} .{ }^{11}$ The endovascular devices and conventional grafts used in this experience are reported in the Appendix E1.

\section{Definition}

Simultaneous intervention defines the treatment of 2 separate aortic lesions (eg, descending thoracic and infrarenal abdominal) during the same operation. Sequential intervention refers to aortic repair of the 2 aortic lesions in 2 separate operations. All variables analyzed in the study were defined according to the Society of Thoracic Surgeons Adult Database definitions. ${ }^{12}$ Morphologic features and outcomes were classified according to the Society for Vascular Surgery/American Association for Vascular Surgery reporting standards. ${ }^{13}$ The extent of the thoracic aortic disease was classified as type A, B, or C as described by Estrera and colleagues. ${ }^{14}$ The proximal landing zone of the thoracic endograft was defined following the "arch map" classification. ${ }^{15}$ Grading the aortic arch atheroma was defined accordingly to a modified classification. ${ }^{16}$ SCI was classified accordingly to the American Spinal Injury Association (ASIA) classification. ${ }^{17}$

\section{Data Analysis}

Clinical data were prospectively recorded and tabulated with Microsoft Excel (Microsoft Corp, Redmond, Wash). Continuous variables were compared between groups with the Mann-Whitney $U$ test or Kruskal-Wallis test, and Fisher exact tests were used in case of dichotomous variables. A stepwise logistic regression model was developed to identify variables associated with SCI development. The stepwise approach was confirmed by backward and forward methods. The significance within the models was evaluated with the Wald test, and the strength of the association of variables with postoperative delirium was estimated by calculating the odds ratio (OR) and $95 \%$ confidence intervals (CIs). The model was calibrated by the Hosmer-Lemeshow goodness-of-fit test and residual diagnostics (deviance and degrees of freedom of $\beta$ ). The discrimination of the model was obtained by calculating the area under the receiver operating characteristic curve. Survivals were estimated by means of the Kaplan-Meier method, and log-rank test was performed for the SCI mortality rate comparison. Results are expressed as mean \pm standard deviation for continuous variables and absolute number with frequencies for the categoric variables. Statistical analysis was computed with SPSS, release 20.0 for Windows (IBM SPSS Inc, Chicago, Ill).

\section{RESULTS \\ General Population}

In the examined period, 4320 patients were treated for thoracic and abdominal aortic diseases. MAD was detected in 77 patients $(1.8 \%)$, of whom $45(58.4 \%)$ were diagnosed simultaneously (Figure 1). Thoracic aortic diseases included degenerative atherosclerotic aneurysms $(n=53$, $68.8 \%$ ), chronic dissecting aneurysm of the descending aorta $(\mathrm{n}=14,18.1 \%)$, and penetrating aortic ulcer $(\mathrm{n}=10,12.9 \%)$. Simultaneous repair was performed in 32 patients $(41.5 \%)$ : In this group, 15 patients $(19.5 \%)$ had total endovascular treatment (Figure 2). Sequential repair was performed in 45 patients (58.4\%): In this group, 9 patients $(11.7 \%)$ had total endovascular repair. In the sequential group, the median interval time between the 2 operations was 182 weeks (range, 2-528 weeks). Interventions were as follows: sequential hybrid repair $(\mathrm{n}=34$, $44.1 \%)$, simultaneous hybrid $(\mathrm{n}=17,22.1 \%)$, simultaneous total endovascular repair $(\mathrm{n}=15,19.5 \%)$, and sequential endovascular $(\mathrm{n}=11,14.3 \%)$. CSFD was placed in 27 patients $(35.0 \%)$. Endograft deployment was

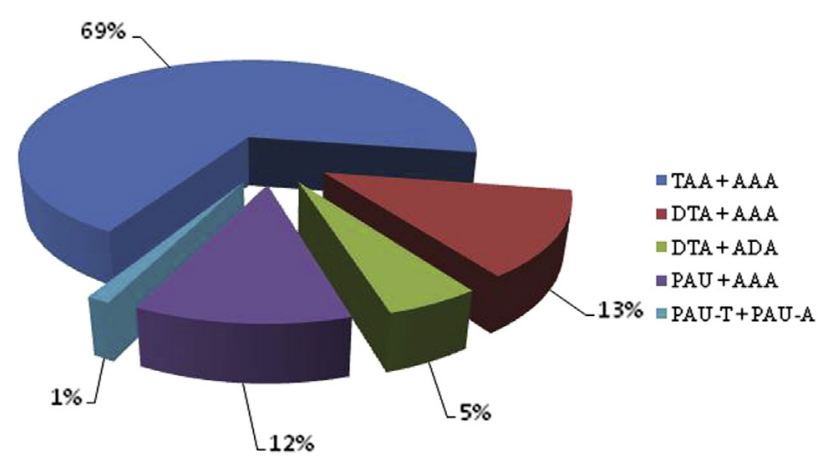

FIGURE 1. Combinations of aortic pathologies that were treated for multilevel aortic repair. $A A A$, Abdominal aortic aneurysm; $A D A$, abdominal dissecting aneurysm; $D T A$, thoracic dissecting aneurysm; $P A U$, penetrating atherosclerotic ulcer; $P A U-A$, abdominal penetrating aortic ulcer; $P A U-T$, thoracic penetrating aortic ulcer; TAA, thoracic aortic aneurysm. 


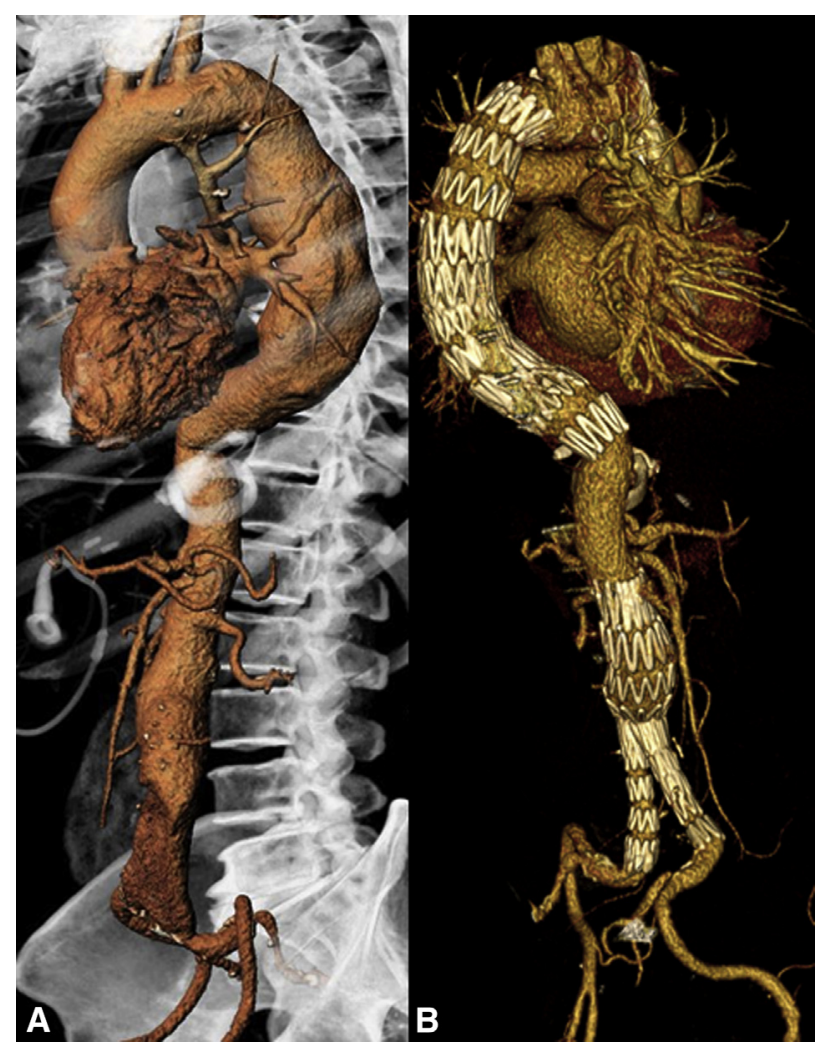

FIGURE 2. Preliminary CT-angiography (A) showing the simultaneous extent type $\mathrm{C}$ thoracic aortic aneurysm and infrarenal aortic aneurysm. Follow-up CT-angiography (B) after total endovascular treatment.

technically successful in all areas. Intraoperative mortality was not observed, and conversion to open repair was never required. Forty-four patients $(57.1 \%)$ were admitted to the intensive care unit. There were no complications at the site of CSFD insertion; no cerebral hemorrhagic complication was observed.

\section{Spinal Cord Ischemia}

Postoperative SCI developed in 6 patients $(7.8 \%)$ (Table 1). Neurologic injury was ASIA grade $\mathrm{C}$ in 4 patients $(5.2 \%)$ and grade A or B in 1 patient $(1.3 \%)$ each. Four $(5.2 \%)$ SCIs resolved (all ASIA grade C), and 2 cases
$(2.6 \%)$ (ASIA grade A and B) were permanent injuries. There were no significant differences between patients with SCI and patients without SCI in terms of comorbidities and operative risk profile (Table 2). No differences were noted for the characteristics of aortic disease, for thoracic or abdominal location, although patients with SCI had a slightly higher prevalence of dissection in the abdominal aorta $(33 \%$ vs $6 \%, P=.051)$. The distance of the distal aortic neck from the celiac trunk was the only significant variable associated with SCI development $(2.5 \pm 2.3 \mathrm{~cm}$ vs $7.4 \pm 5.9 \mathrm{~cm}, P=.038$ ). In regard to technical and operative data, CSFD, LSA coverage, and preventive LSA revascularization did not influence SCI occurrence ( $P=.659, P=.999$, and $P=.494$, respectively $)$ Likewise, operative strategies were not SCI related (OR, 1.2; 95\% CI, 0.50-2.86; $P=.688$ ) (Table 3). On multivariate analysis, the distance from the celiac trunk was the only independent predictor of postoperative SCI (OR, 0.75; 95\% CI, 0.56-0.99; $P=.046$ ); open surgical repair of the abdominal aortic aneurysm was associated with a higher risk of SCI but did not reach statistical significance (OR, 0.16; 95\% CI, 0.02-1.06; $P=.057$ ) (Table 4). With all patients considered, the variables of the model correctly predicted $92 \%$ of the observed cases of postoperative SCI. The area under the receiver operating characteristic curve for this model was .856, suggesting a good ability to distinguish between individuals in whom SCI will develop and those in whom SCI will not develop. The Hosmer-Lemeshow goodness-of-fit test was not significant for lack of fit (chi-square [8df] $=7.75, P=.458$ ), indicating that there was no statistically significant departure from a perfect fit. Figure 3 displays the probability of SCI occurrence in relation to the distance from celiac trunk and type of approach to abdominal region.

\section{Outcomes and Survival}

Operative mortality was $9.1 \%(n=7)$. Although patients with SCI had a 2-fold risk of operative mortality, it was not statistically significant $(17 \%$ vs $9 \%, P=.447)$. Patients with SCI had a longer intensive care unit stay ( \pm \pm 5 days vs $3 \pm 14$ days, $P=.006)$ and a longer, although not significantly different, hospitalization $(21 \pm 14$ days vs $14 \pm 15$

TABLE 1. Summary of procedural details and neurologic deficits of 6 patients with postoperative spinal cord injury

\begin{tabular}{|c|c|c|c|c|c|c|c|c|c|c|c|}
\hline Patient & Intervention & $\begin{array}{c}\text { Thoracic } \\
\text { disease }\end{array}$ & $\begin{array}{l}\text { Delay } \\
\text { (wk) }\end{array}$ & $\begin{array}{c}\text { LSA } \\
\text { coverage }\end{array}$ & $\begin{array}{c}\text { Aortic } \\
\text { coverage }(\mathbf{c m})\end{array}$ & $\begin{array}{c}\text { CT } \\
\text { distance }(\mathrm{mm})\end{array}$ & $\begin{array}{c}\text { AAA } \\
\text { procedure }\end{array}$ & CSFD & $\begin{array}{c}\text { Neurologic } \\
\text { injury* }\end{array}$ & Onset & Recovery \\
\hline$\# 1$ & Sequential & TAA & 56 & No & C (35) & 6.0 & EVAR & No & Grade A & $<6 \mathrm{~h}$ & No \\
\hline$\# 2$ & Simultaneous & Ulcer & 0 & No & A (10) & 5.4 & EVAR & No & Grade C & $>6 \mathrm{~h}$ & Yes \\
\hline \#3 & Sequential & DTA & 6 & Yes & B (15) & 0 & EVAR & Yes & Grade B & $<6 \mathrm{~h}$ & No \\
\hline \#4 & Sequential & DTA & 172 & No & C (30) & 0 & EVAR & No & Grade C & $<6 \mathrm{~h}$ & Yes \\
\hline \#5 & Simultaneous & TAA & 0 & No & C (25) & 2.0 & Open & Yes & Grade C & $<6 \mathrm{~h}$ & Yes \\
\hline \#6 & Simultaneous & TAA & 0 & No & B (15) & 2.4 & Open & Yes & Grade C & $<6 \mathrm{~h}$ & Yes \\
\hline
\end{tabular}

$A A A$, Abdominal aortic aneurysm; CSFD, cerebrospinal fluid drainage; $C T$, celiac trunk; DTA, dissecting thoracic aortic aneurysm; EVAR, endovascular aortic repair; $L S A$, left subclavian artery; TAA, thoracic aortic aneurysm. *Severity grade according to American Spinal Injury Association. 
TABLE 2. Demographic data, risk factors, and operative outcomes

\begin{tabular}{|c|c|c|c|c|}
\hline Variable* & $\begin{array}{c}\text { All patient } \\
\mathbf{n}=\mathbf{7 7}\end{array}$ & $\begin{array}{c}\text { No SCI } \\
\mathbf{n}=\mathbf{7 1}\end{array}$ & $\begin{array}{c}\mathrm{SCI} \\
\mathrm{n}=\mathbf{7 2}\end{array}$ & $\begin{array}{r}P \\
\text { value }\end{array}$ \\
\hline \multicolumn{5}{|l|}{ Demographic } \\
\hline Age, y & $72 \pm 8$ & $72 \pm 7$ & $66 \pm 14$ & .093 \\
\hline Gender (male) & $70(91)$ & $65(92)$ & $5(83)$ & .447 \\
\hline \multicolumn{5}{|l|}{ Comorbidities } \\
\hline CAD & $27(35)$ & $26(37)$ & $1(17)$ & .417 \\
\hline LVEF $<25 \%$ & $3(4)$ & $3(4)$ & $0(0)$ & .999 \\
\hline Arrhythmia & $21(27)$ & $21(30)$ & $0(0)$ & .181 \\
\hline Hypertension & $71(92)$ & $65(92)$ & $6(100)$ & .999 \\
\hline Diabetes & $12(16)$ & $11(16)$ & $1(17)$ & .999 \\
\hline Dyslipidemia & $45(58)$ & $41(58)$ & $4(67)$ & .999 \\
\hline COPD & $38(49)$ & $37(52)$ & $1(17)$ & .200 \\
\hline CVA & $8(10)$ & $8(11)$ & $0(0)$ & .999 \\
\hline $\mathrm{eGFR}<30 \mathrm{~mL} / \mathrm{min} / 1.73 \mathrm{~m}^{2}$ & $5(7)$ & $5(7)$ & $0(0)$ & .622 \\
\hline \multicolumn{5}{|l|}{ Thoracic aorta } \\
\hline \multicolumn{4}{|l|}{ Pathology } & .542 \\
\hline Atherosclerotic & $53(69)$ & $50(70)$ & $3(50)$ & \\
\hline Dissection & $14(18)$ & $12(17)$ & $2(33)$ & \\
\hline Penetrating ulcer & $10(13)$ & $9(13)$ & $1(17)$ & \\
\hline Diameter $(\mathrm{mm} \pm \mathrm{SD})$ & $61 \pm 18$ & $61 \pm 18$ & $55 \pm 21$ & .435 \\
\hline \multicolumn{4}{|l|}{ Thoracic disease extent } & .398 \\
\hline Type A & $17(22)$ & $17(24)$ & $0(0)$ & \\
\hline Type B & $30(39)$ & $27(38)$ & $3(50)$ & \\
\hline Type C & $30(39)$ & $27(38)$ & $3(50)$ & \\
\hline \multicolumn{4}{|l|}{ Arch atheroma } & .774 \\
\hline Grade 0 & $2(3)$ & $2(3)$ & $0(0)$ & \\
\hline Grade 1 & $50(65)$ & $45(63)$ & $5(83)$ & \\
\hline Grade 2 & $21(27)$ & $20(28)$ & $1(17)$ & \\
\hline Grade 3 & $4(5)$ & $4(6)$ & $0(0)$ & \\
\hline $\begin{array}{l}\text { Distance from CT } \\
\quad(\mathrm{mm} \pm \mathrm{SD})\end{array}$ & $7 \pm 6$ & $7 \pm 6$ & $3 \pm 2$ & .038 \\
\hline \multicolumn{5}{|l|}{ Abdominal aorta } \\
\hline \multicolumn{4}{|l|}{ Pathology } & .051 \\
\hline Atherosclerotic & $70(90)$ & $66(93)$ & $4(67)$ & \\
\hline Dissection & $6(8)$ & $4(6)$ & $2(33)$ & \\
\hline Inflammatory & $1(1)$ & $1(1)$ & $0(0)$ & \\
\hline Diameter $(\mathrm{mm} \pm \mathrm{SD})$ & $60 \pm 11$ & $60 \pm 11$ & $56 \pm 4$ & .381 \\
\hline \multicolumn{5}{|l|}{ Intraoperative details } \\
\hline \multicolumn{4}{|l|}{ Timing } & .688 \\
\hline Simultaneous & $32(39)$ & $29(41)$ & $3(50)$ & \\
\hline Sequential & $45(58)$ & $42(59)$ & $3(50)$ & \\
\hline \multicolumn{4}{|l|}{ Proximal landing zone } & .579 \\
\hline “zone 0" & $4(6)$ & $4(6)$ & $0(0)$ & \\
\hline "zone $1 "$ & $3(4)$ & $3(4)$ & $0(0)$ & \\
\hline "zone $2 "$ & $10(13)$ & $8(11)$ & $2(33)$ & \\
\hline "zone 3" & $35(46)$ & $33(47)$ & $2(33)$ & \\
\hline "zone 4" & $25(33)$ & $23(32)$ & $2(33)$ & \\
\hline CSFD & $27(35)$ & $24(34)$ & $3(50)$ & .659 \\
\hline LSA coverage & $17(22)$ & $16(23)$ & $1(17)$ & .999 \\
\hline LSA revascularization & $8(10)$ & $7(10)$ & $1(17)$ & .494 \\
\hline $\begin{array}{l}\text { Aortic coverage } \\
\qquad(\mathrm{mm} \pm \mathrm{SD})\end{array}$ & $200 \pm 85$ & $201 \pm 84$ & $217 \pm 98$ & .694 \\
\hline $\mathrm{EG}$ diameter $(\mathrm{mm} \pm \mathrm{SD})$ & $38 \pm 4$ & $38 \pm 4$ & $37 \pm 4$ & .651 \\
\hline EG implanted $(\mathrm{n} \pm \mathrm{SD})$ & $1.5 \pm 0.7$ & $1.5 \pm 0.7$ & $1.5 \pm 0.6$ & .897 \\
\hline Open abdominal repair & $51(66)$ & $49(69)$ & $2(33)$ & .171 \\
\hline
\end{tabular}

TABLE 2. Continued

\begin{tabular}{|c|c|c|c|c|}
\hline Variable* & $\begin{array}{c}\text { All patient } \\
\mathbf{n}=77\end{array}$ & $\begin{array}{l}\text { No SCI } \\
n=71\end{array}$ & $\begin{array}{c}\text { SCI } \\
\mathrm{n}=\mathbf{7 2}\end{array}$ & $\begin{array}{c}P \\
\text { value }\end{array}$ \\
\hline \multicolumn{5}{|l|}{ Postoperative details } \\
\hline $\mathrm{ICU}(\mathrm{d} \pm \mathrm{SD})$ & $4 \pm 13$ & $6 \pm 18$ & $9 \pm 4$ & .002 \\
\hline ICU admission & $44(57)$ & $39(55)$ & $5(83)$ & .230 \\
\hline $\operatorname{LOS}(\mathrm{d} \pm \mathrm{SD})$ & $15 \pm 15$ & $14 \pm 15$ & $21 \pm 14$ & .078 \\
\hline Hospital mortality & $7(9)$ & $6(9)$ & $1(17)$ & .447 \\
\hline \multicolumn{5}{|c|}{$\begin{array}{l}C A D \text {, Coronary artery disease; } C O P D \text {, chronic obstructive pulmonary disease; } \\
C S F D \text {, cerebrospinal fluid drain; } C T \text {, celiac trunk; } C V A \text {, cerebrovascular accident; } \\
E G \text {, endograft; } e G F R \text {, estimated glomerular filtration rate; } I C U \text {, intensive care unit; } \\
L O S \text {, length of stay; } L S A \text {, left subclavian artery; } L V E F \text {, left ventricular ejection } \\
\text { fraction; } S C I \text {, spinal cord ischemia; } S D \text {, standard deviation. *Data for continuous } \\
\text { variables are shown as mean SD, and data for categoric variables are shown as } \\
\text { number }(\%) \text {. }\end{array}$} \\
\hline
\end{tabular}

days, $P=.078$ ). Finally, the median follow-up was 15 months (range, 1-108 months). During the follow-up, type 1 endoleak was detected in 8 patients $(11.4 \%)$. Five $(7.1 \%)$ thoracic distal type 1 endoleaks were successfully treated with an extender. Two $(2.8 \%)$ abdominal type 1 endoleaks (proximal $\mathrm{n}=1$, distal $\mathrm{n}=1$ ) resolved with an extender. Two (2.8\%) abdominal type 2 endoleaks resolved after coil embolization $(n=1)$ or spontaneously thrombosed $(\mathrm{n}=1)$. Actuarial survival estimates at 1,2 , and 5 years after the procedure were $80 \% \pm 5 \%, 68 \% \pm 6 \%$, and $63 \% \pm 7 \%$, respectively (Figure 4); survival was not negatively impaired by SCI occurrence $(P=.885)$.

\section{DISCUSSION}

No comprehensive data are available on the real incidence of MAD, and guidelines for the appropriate treatment strategy are missing. The first potential

TABLE 3. Operative strategy analysis

\begin{tabular}{|c|c|c|c|}
\hline Variable* & $\begin{array}{c}\text { Simultaneous } \\
(\mathbf{n}=\mathbf{3 2})\end{array}$ & $\begin{array}{c}\text { Sequential } \\
(n=44)\end{array}$ & $\begin{array}{c}P \\
\text { value }\end{array}$ \\
\hline \multicolumn{4}{|l|}{ Demographic $(\%)$} \\
\hline Age $( \pm$ SD $)$ & $69.9 \pm 8.3$ & $72.9 \pm 6.8$ & .121 \\
\hline Gender (male) & $28(87.5)$ & $42(93.3)$ & .441 \\
\hline Thoracic disease extent $(\%)$ & & & .026 \\
\hline Type A & $4(12.5)$ & $13(28.9)$ & \\
\hline Type B & $18(56.2)$ & $12(26.7)$ & \\
\hline Type C & $10(31.2)$ & $20(44.4)$ & \\
\hline Distance from CT $(\mathrm{mm} \pm \mathrm{SD})$ & $7.5 \pm 6.7$ & $6.3 \pm 4.4$ & .820 \\
\hline \multicolumn{4}{|l|}{ Operative details (\%) } \\
\hline Covered aorta $(\mathrm{mm} \pm \mathrm{SD})$ & $170.3 \pm 70.5$ & $224.6 \pm 87.5$ & .004 \\
\hline CSFD & $16(50.0)$ & $11(24.4)$ & .021 \\
\hline LSA coverage & $2(6.2)$ & $15(33.3)$ & .005 \\
\hline LSA revascularization & $0(0)$ & $8(17.8)$ & .018 \\
\hline \multicolumn{4}{|l|}{ Outcomes $(\%)$} \\
\hline In-hospital mortality & $3(9.4)$ & $4(8.9)$ & .942 \\
\hline SCI & $3(9.4)$ & $3(6.7 \%)$ & .688 \\
\hline
\end{tabular}


TABLE 4. Predictors of spinal cord injury: Univariate and multivariate analyses

\begin{tabular}{lcccc}
\hline \multicolumn{1}{c}{ Variables } & $\begin{array}{c}\text { Univariate } \\
\text { OR }(\mathbf{9 5} \% \mathbf{C I})\end{array}$ & $\begin{array}{c}\boldsymbol{P} \\
\text { value }\end{array}$ & $\begin{array}{c}\text { Multivariate } \\
\text { OR }(\mathbf{9 5} \% \mathbf{C I})\end{array}$ & $\begin{array}{c}\boldsymbol{P} \\
\text { value }\end{array}$ \\
\hline Age (y) & $0.92(0.84-1.01)$ & .093 & & \\
Arrhythmia & $0.89(0.82-0.98)$ & .181 & & \\
COPD & $0.18(0.20-1.65)$ & .200 & & \\
Distance from CT (mm) & $0.78(0.59-1.01)$ & .038 & $0.75(0.56-0.99)$ & .046 \\
Abdominal pathology* & $3.39(0.69-16.61)$ & .051 & & \\
Open abdominal repair & $0.22(0.04-1.32)$ & .171 & $0.78(0.02-1.06)$ & .057 \\
\hline
\end{tabular}

$C I$, Confidence interval; $C O P D$, chronic obstructive pulmonary disease; $C T$, celiac trunk; $O R$, odds ratio. *Atherosclerotic abdominal disease versus dissection and inflammatory.

explanation may lie in the fact that MAD is an uncommon finding. ${ }^{1-4}$ We did not perform a specific analysis on the incidence of MAD, but our results are in accordance with the literature. The $1.8 \%$ rate of multilevel aortic repair in our study is similar to the $3.4 \%$ reported by Gloviczki and colleagues, ${ }^{2}$ confirming that treatment for MAD accounts for a small part of all aortic aneurysm interventions. Another similarity with the literature is the incidence ratio of simultaneous and sequential aortic lesions. In our experience, $58.4 \%$ of the cases were recognized before the first operation, data that are close to the $46 \%$ to $54 \%$ reported in previous published series. ${ }^{2,3}$

Uncertainty around MAD treatment strategy has been generated by the conflicting data published so far (Table 5). ${ }^{5-9,18-22}$ Two recent articles showed that TEVAR could increase the occurrence of SCI in patients with a history of abdominal aortic aneurysm repair by approximately 7 - to 14 -fold. ${ }^{5,6}$ On the contrary, Martin and colleagues ${ }^{7}$ reported that a prior abdominal aortic aneurysm repair, per se, was not significantly associated with SCI. An interim analysis of the European Registry of Endovascular Aortic Repair Complications added uncertainty. ${ }^{23}$ In that study, SCI was associated with the simultaneous closure of at least 2 independent vascular territories supplying the spinal cord, but previous closure of a single vascular territory was not associated with an increased risk of symptomatic SCI. An interesting point of our study is the comparison of simultaneous and sequential treatment of MAD. Although we used adjuncts, the $7.8 \%$ overall rate of SCI is still disappointedly high but not outside the reported range for an isolated TEVAR. ${ }^{24}$ Because of the heterogeneity of MAD presentation and the multiple aspects involved in every type of treatment, our work does not have the presumption to indicate definitively the superiority of one treatment over the other. The reason why simultaneous and sequential MAD treatment had no significantly different SCI rate might be explained in 2 different ways: on the one hand, the hypothetical increased risk of the simultaneous treatment of the 2 districts can benefit from the rapidity and minimally invasiveness of TEVAR, especially for less-extensive aortic coverage. On the other hand, although sequential repair required a more extensive aortic coverage, spinal cord blood flow might have benefited from the recruitment of collaterals over time. $^{25}$

This hypothesis deserves further consideration. Etz and colleagues $^{26}$ showed that SCI may occur less frequently when the repair is performed in a 2-stage approach rather than a single procedure despite a higher number of intercostal arteries sacrificed. This experience included open repair only, but Johnston and colleagues ${ }^{27}$ obtained the same results using a hybrid operation, taking advantage of a TEVAR-first approach that was subsequently completed with an abdominal open graft repair. On the basis of these observations, if a sequential repair is advocated as

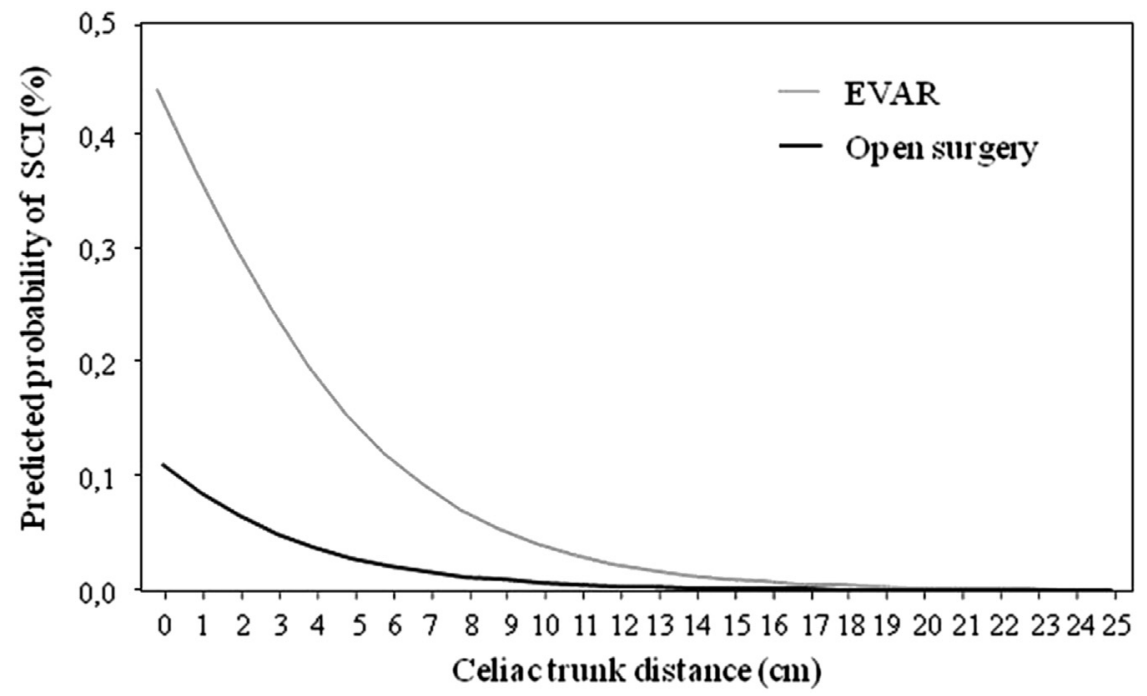

FIGURE 3. Probability of SCI occurrence in relation to the distance of the distal neck from celiac trunk and type of abdominal aortic repair. EVAR, Endovascular repair; SCI, spinal cord injury. 


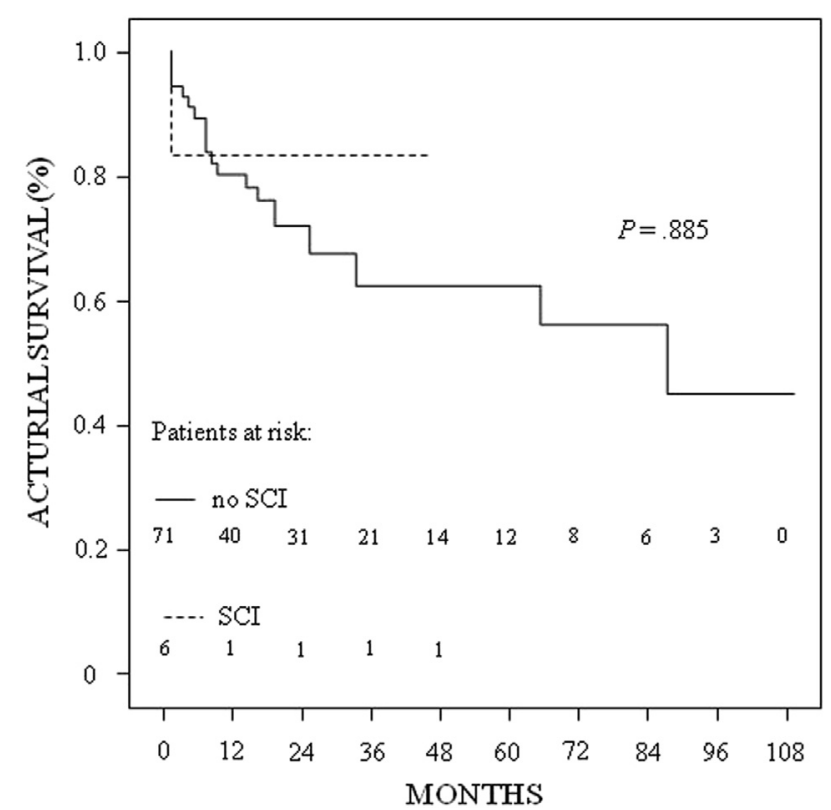

FIGURE 4. Kaplan-Meier estimates of survival. SCI, Spinal cord injury.

the best strategy for the treatment of MAD, it should be equally noted that an increased interval time among 2 aortic interventions has been found to be predictive of SCI. ${ }^{10}$ It is disturbing that in our sequential interventions, 3 cases of SCI developed in sporadic time intervals. Although MAD is a noncontinuous disease, it could be at increased risk for SCI similar to the thoracoabdominal extent; therefore, it would be more useful to identify the ideal time interval between the 2 operations when MAD is planned to be repaired sequentially.

The length of aortic coverage has been reported as a predictive factor of SCI; however, definitive consensus on that has not been reached so far. ${ }^{7,28,29}$ We expected the length of aortic coverage would play a role in SCI development, but in our experience the only determinant of SCI was the distance of the landing zone from the celiac trunk. The "collateral network" concept showed intercostal artery occlusion may not be sufficient per se to induce SCI, but those intercostals between $T_{8}$ and $L_{2}$ play a critical role in spinal perfusion. ${ }^{8,25,28}$ Although Amabile and colleagues ${ }^{30}$ found aortic coverage to be predictive of $\mathrm{SCI}$, they were able to hypothesize only a greater risk of SCI with the coverage of the distal third of the thoracic aorta.
Our analysis confirms the importance of this anatomic aspect. By analyzing our SCI cases, we observe this event occurred only after an extent type B or C coverage. Even in the TEVAR era, the involvement of the most distal segment of the descending thoracic aorta in MAD is at a higher risk of SCI development. Another interesting observation comes from the experience of Takagi and colleagues. $^{31}$ They did not report SCI in patients with proximal thoracic aortic repair, whereas their only case of paraplegia occurred in the descending group despite the reattachment of the seventh and eighth intercostal arteries. With all these aspects considered, we speculate that simultaneous repair of MAD might be more suitable in case of extent type A aortic lesion, whereas distal descending replacements should be approached with caution. $^{30}$

To date, only 1 article has reported a comparison of the type of MAD treatment in which an endograft is used in 1 or both aortic segments. Aguiar Lucas and colleagues ${ }^{8}$ published the results of 38 MAD cases and did not observe SCI with hybrid or totally endovascular repair, with a simultaneous or sequential strategy. The SCI rate of hybrid and total endovascular strategy also did not differ significantly in our experience, but we did observe some cases of SCI. In view of the predictor of SCI identified in our analysis, these data may be justified by the fact that in our series the most distal segment of the descending aorta was involved 4 times more frequently than in their experience.

Perioperative adjuncts have been associated with improved neurologic outcomes after open conventional thoracic aortic repair. ${ }^{32}$ Although CSFD and LSA bypass have been recognized as important adjuncts to limit the risk of SCI, there are no definitive statements regarding their mandatory use during TEVARs. ${ }^{32}$ Hnath and colleagues ${ }^{33}$ prospectively evaluated their standardized protocol of CSFD during TEVAR and showed that selective use may offer the same benefit as mandatory drainage. Similarly for LSA management, in the experience of Maldonado and colleagues, ${ }^{34}$ prior aortic repair did not seem to be a predictor of SCI when a strategy of selective revascularization was adopted. Our SCI rate was not irrelevant, even in this endovascular era. However, we should remember that in contrast to that study, our cohort inherently was at a higher risk of SCI. Admittedly, our

TABLE 5. Multilevel aortic disease treatment: Literature summary

\begin{tabular}{lccccc}
\hline \multicolumn{1}{c}{ Authors } & Year & Treatment (Simultaneous/sequential) & SCI $(\%)$ & Permanent $(\%)$ & Predictors \\
\hline Baril and colleagues $^{5}$ & 2006 & $1 / 27$ & 14.3 & 10.7 & NSR \\
Martin and colleagues $^{7}$ & 2009 & $0 / 51$ & 26.0 & NSR & History of AAA repair \\
Schlösser and colleagues $^{6}$ & 2009 & $0 / 72$ & 12.5 & 6.9 & LSA coverage \\
Aguiar Lucas and colleagues $^{8}$ & 2009 & $3 / 36$ & 0 & 0 & \\
Scali and colleagues $^{10}$ & 2012 & $22 / 0$ & 13.6 & 4.5 & Renal insufficiency \\
\hline
\end{tabular}

$A A A$, Abdominal aortic aneurysm; $L S A$, left subclavian artery; $N S R$, not specifically reported; $S C I$, spinal cord injury. 
selective approach of LSA bypass and CSFD can mask their real clinical impact. It is also true that using a selected approach has allowed us to have similar risks in conditions at greater risk for SCI.

Long-term results have not been widely reported in MAD series. In their remarkable experience, Crawford and Cohen $^{1}$ reported that long-term survival was better in patients with complete aortic treatment because most of the early deaths in the sequential intervention were caused by rupture of the second aneurysm. ${ }^{2,4}$ On the other hand, Gloviczki and colleagues ${ }^{2}$ showed improved survival at 3 and 5 years after sequential repair, although it was not statistically significant. Few current series have reported long-term follow-up for MAD: Moon and colleagues ${ }^{4}$ published actuarial survival estimates of $87 \%$ at 1 year and $81 \%$ at 3 years, but the mean follow-up was only 13.2 months. Scali and colleagues ${ }^{10}$ demonstrated an overall survival of $81 \%$ at 1 year for simultaneous totally endovascular repair. Our data show results comparable to those of older reports on MAD; our overall $63 \%$ survival at 5 years reflects the significant impact of such an extensive disease on survival is similar to the reported long-term survival of thoracoabdominal aortic diseases. ${ }^{35}$

\section{Study Limitations}

The multicenter design of this study has several limitations. The sample size of the 2 groups remains small for adequate analyses. The rarity of this condition makes a large and homogeneous study difficult to be realized. Another important limitation was the retrospective design of the study. The recruiting centers had significant expertise and experience in open and endovascular treatment of aortic pathologies, but selection bias may have occurred. Third, our data were prospectively collected and analyses finally identified a significant predictor, but potential confounding variables may have affected the results. Furthermore, the absence of guidelines for the management of MAD makes the study heterogeneous in treatment strategy. Despite these limitations, a similar design is present in other studies and our cohort is currently one of the largest available series, offering new sources of debate..$^{5,7}$

\section{CONCLUSIONS}

Our study supports previous findings that MAD is an uncommon clinical entity, either in terms of the incidence rate or the impact on overall aortic interventions. Despite the best efforts to refine treatment strategy and the use of technical adjuncts, the risk of SCI is still substantial at $8 \%$ in patients with MAD, even in this endovascular era. In our experience, the distance of the distal landing zone from the celiac trunk was the only predictive factor of SCI. This means the coverage of the distal descending thoracic aorta in MAD is at a higher risk of SCI.
Long-term survival results are satisfactory and seem to be comparable to those of thoracoabdominal aortic diseases.

\section{References}

1. Crawford ES, Cohen ES. Aortic aneurysm: a multifocal disease. Arch Surg. 1982; 117:1393-400

2. Gloviczki P, Pairolero P, Welch T, Cherry K, Hallett J, Toomey B, et al. Multiple aortic aneurysms: the results of surgical management. J Vasc Surg. 1990;11:19-27.

3. Chaer RA, Vasoncelos R, Marone LK, Al-Khoury G, Rhee RY, Cho JS, et al Synchronous and metachronous thoracic aneurysms in patients with abdominal aortic aneurysms. J Vasc Surg. 2012;56:1261-5.

4. Moon MR, Mitchell RS, Dake MD, Zarins CK, Fann JI, Miller DC. Synchronous abdominal aortic replacement and thoracic stent-graft placement for multilevel aortic disease. J Vasc Surg. 1997;25:332-40.

5. Baril DT, Carroccio A, Ellozy SH, Palchik E, Addis MD, Jacobs TS, et al. Endovascular thoracic aortic repair and previous or concomitant abdominal aortic repair: is the increased risk of spinal cord ischemia real? Ann Vasc Surg. 2006;20:188-94.

6. Schlösser FJ, Verhagen HJ, Lin PH, Verhoeven EL, van Herwaarden JA, Moll FL, et al. TEVAR following prior abdominal aortic aneurysm surgery: increased risk of neurological deficit. J Vasc Surg. 2009;49:308-14.

7. Martin DJ, Martin TD, Hess PJ, Daniels MJ, Feezor RJ, Lee WA. Spinal cord ischemia after TEVAR in patients with abdominal aortic aneurysms. $J$ Vasc Surg. 2009;49:302-6.

8. Aguiar Lucas L, Rodriguez-Lopez JA, Olsen DM, Diethrich EB. Endovascular repair in the thoracic and abdominal aorta: no increased risk of spinal cord ischemia when both territories are treated. J Endovasc Ther. 2009;16:189-96.

9. Ullery BW, Cheung AT, Fairman RM, Jackson BM, Woo EY, Bavaria J, et al. Risk factors, outcomes, and clinical manifestations of spinal cord ischemia following thoracic endovascular aortic repair. J Vasc Surg. 2011;54:677-84.

10. Scali ST, Feezor RJ, Chang CK, Stone DH, Goodney PP, Nelson PR, et al. Safety of elective management of synchronous aortic disease with synchronous thoracic and aortic stent graft placement. J Vasc Surg. 2012;56:957-64.

11. Cheung AT, Pochettino A, McGarvey ML, Appoo JJ, Fairman RM, Carpenter JP et al. Strategies to manage paraplegia risk after endovascular stent repair of descending thoracic aortic aneurysms. Ann Thorac Surg. 2005;80:1280-8.

12. Clarck RE. Committee to develop a national database for thoracic surgeons Definitions of terms of the Society of Thoracic Surgeons national cardiac surgery database. Ann Thorac Surg. 1994;58:271-3.

13. Chaikof EL, Fillinger MF, Matsumura JS, Rutherford RB, White GH Blankensteijn JD, et al. Identifying and grading factors that modify the outcome of endovascular aortic aneurysm repair. J Vasc Surg. 2002;35:1061-6.

14. Estrera AL, Miller CC 3rd, Chen EP, Meada R, Torres RH, Porat EE, et al Descending thoracic aortic aneurysm repair: 12-year experience using distal aortic perfusion and cerebrospinal fluid drainage. Ann Thorac Surg. 2005;80: 1290-6.

15. Criado FJ, Clark NS, Barnatan MF. Stent graft repair in the aortic arch and descending thoracic aorta: a 4-year experience. J Vasc Surg. 2002;36:1121-8.

16. Gutsche JT, Cheung AT, McGarvey ML, Moser WG, Szeto W, Carpenter JP, et al Risk factors for perioperative stroke after thoracic endovascular aortic repair Ann Thorac Surg. 2007;84:1195-200.

17. American Spinal Injury Association. Reference Manual of the International Standards for Neurological Classification of Spinal Cord Injury. Chicago, IL: American Spinal Injury Association; 2003.

18. Meguid AA, Bove PG, Long GW, Kirsch MJ, Bendick PJ, Zelenock GB Synchronous stent-graft repair of thoracic and infrarenal abdominal aortic aneurysms. J Endovasc Ther. 2002;9:165-9.

19. Szmidt J, Rowiński O, Gałazka Z, Jakimowicz T, Nazarewski S, Grochowiecki T, et al. Synchronous endovascular exclusion of thoracic aortic aneurysm with open abdominal aortic aneurysm repair. Eur J Vasc Endovasc Surg. 2004;28: 442-8.

20. Kawaharada N, Morishita K, Kurimoto Y, Hyodoh H, Ito T, Harada R, et al. Spinal cord ischemia after elective endovascular stent-graft repair of the thoracic aorta. Eur J Cardiothorac Surg. 2007;31:998-1003.

21. Kirkwood ML, Pochettino A, Fairman RM, Jackson BM, Wang GJ, Szeto WY, et al. Synchronous thoracic endovascular aortic repair and endovascular aortic repair is feasible with minimal morbidity and mortality. J Vasc Surg. 2011;54 1588-91. 
22. Ullery BW, Wang GJ, Woo EY, Cheung AT, McGarvey ML, Carpenter JP, et al. No increased risk of spinal cord ischemia in delayed AAA repair following thoracic aortic surgery. Vasc Endovascular Surg. 2013;47:85-91.

23. Czerny M, Eggebrecht H, Sodeck G, Verzini F, Cao P, Maritati G, et al. Mechanisms of symptomatic spinal cord ischemia after TEVAR: insights from the European Registry of Endovascular Aortic Repair Complications (EuREC). J Endovasc Ther. 2012;19:37-43.

24. Rizvi AZ, Sullivan TM. Incidence, prevention, and management in spinal cord protection during TEVAR. J Vasc Surg. 2010;52(4 Suppl):86S-90S.

25. Griepp RB, Griepp EB. Spinal cord perfusion and protection during descending thoracic and thoracoabdominal aortic surgery: the collateral network concept. Ann Thorac Surg. 2007;83:S865-9.

26. Etz CD, Zoli S, Mueller CS, Bodian CA, Di Luozzo G, Lazala R, et al. Staged repair significantly reduces paraplegia rate after extensive thoracoabdominal aortic aneurysm repair. J Thorac Cardiovasc Surg. 2010;139:1464-72.

27. Johnston WF, Upchurch GR Jr, Tracci MC, Cherry KJ, Ailawadi G, Kern JA. Staged hybrid approach using proximal thoracic endovascular aneurysm repair and distal open repair for the treatment of extensive thoracoabdominal aortic aneurysms. J Vasc Surg. 2012;56:1495-502.

28. Greenberg RK, Lu Q, Roselli EE, Svensson LG, Moon MC, Hernandez AV, et al. Contemporary analysis of descending thoracic and thoracoabdominal aneurysm repair: a comparison of endovascular and open techniques. Circulation. 2009; 118:808-17.
29. Feezor RJ, Martin TD, Hess PJ Jr, Daniels MJ, Beaver TM, Klodell CT, et al. Extent of aortic coverage and incidence of spinal cord ischemia after thoracic endovascular aneurysm repair. Ann Thorac Surg. 2008;86: 1809-14.

30. Amabile P, Grisoli D, Giorgi R, Bartoli JM, Piquet P. Incidence and determinants of spinal cord ischaemia in stent-graft repair of the thoracic aorta. Eur J Vasc Endovasc Surg. 2008;35:455-61.

31. Takagi H, Mori Y, Iwata H, Umeda Y, Fukumoto Y, Matsuno Y, et al. Synchronous operations for combined thoracic and abdominal aortic aneurysms. Surg Today. 2003;33:674-8.

32. Keith CJ Jr, Passman MA, Carignan MJ, Parmar GM, Nagre SB, Patterson MA, et al. Protocol implementation of selective postoperative lumbar spinal drainage after thoracic aortic endograft. J Vasc Surg. 2012;55:1-8.

33. Hnath JC, Mehta M, Taggert JB, Sternbach Y, Roddy SP, Kreienberg PB, et al. Strategies to improve spinal cord ischemia in endovascular thoracic aortic repair: outcomes of a prospective cerebrospinal fluid drainage protocol. J Vasc Surg. 2008;48:836-40.

34. Maldonado TS, Dexter D, Rockman CB, Veith FJ, Garg K, Arko F, et al. Left subclavian artery coverage during thoracic endovascular aortic aneurysm repair does not mandate revascularization. J Vasc Surg. 2013;57: 116-24.

35. Conrad MF, Crawford RS, Davison JK, Cambria RP. Thoracoabdominal aneurysm repair: a 20-year perspective. Ann Thorac Surg. 2007;83:S856-61. 


\section{APPENDIX E1}

Thoracic endograft:

Excluder/TAG/C-TAG (WL Gore \& Associates;

Flagstaff, Ariz)

Talent/Valiant/Captivia (Medtronic Vascular, Santa

Rosa, Calif)

Relay (Bolton Medical, Sunrise, Fla)

TX-1/TX-2 (Cook, Bloomington, Ind)

Abdominal endograft:

Excluder/C3 (WL Gore \& Associates)

Talent/Endurant (Medtronic Vascular)

Zenith (Cook)

Conventional abdominal graft:

Uni-graft (B. Braun Medical, Melsungen, Germany)

Intergard (Maquet Gmbh, Rastatt, Germany)

Vascular graft (WL Gore \& Associates) 